\title{
Peripheral neuropathy as the common ground between eastern and western medicine
}

\begin{abstract}
This work concerns the direct connection of Western and Eastern Medicine. Both are based in a greater part upon what modern western medicine calls Peripheral Neuropathy (PN). A review of both $\mathrm{PN}$ and Chinese medicine are delineated and discussed in reference to each other. It is demonstrated that the signs and symptoms of PN are exactly the same as the signs and symptoms of Chinese medicine and thus both have this common ground between them. It is very interesting that Chinese medicine describes the signs and symptoms of PN before the advent of modern physiology.
\end{abstract}

Keywords: peripheral neuropathy, nerve cell disfunction, autoimmune disorders, friedreich's ataxia, guillian-barre' syndrome
Volume 12 Issue 6 - 2019

Edward F Block

Block Institute for Astrobiological Studies, USA

Correspondence: Edward F Block, Intitute for Astrobiological Studies is now: 160 Holiday Loop, Unit 2, New Braunfels, TX 78132, USA, Email edwrd.f.block.iv@gmail.com

Received: October 31, 2019 | Published: November 25, 2019
Abbreviations: PN, peripheral neuropathy; ECM, extracellular matrix; ATP, adenosine tri-phosphate; CIDP, chronic inflammatory demyelinating syndrome; AIDP, acute inflammatory demyelinating polyneuropathy; TCM, Traditional Chinese medicine; BMR, basal metabolic rate

\section{Introduction}

In my study of both Western and Eastern medicine, I have noticed what I hypothesize to be the common link between these different systems of medical thought. This link deals with the effects within the body of neuropathy, a condition of nerve cell disfunction, caused by physical, emotional \& mental trauma, parasites, bacterial and viral infections, aberrations in diet (including alcoholism), autoimmune disorders, toxins and environmental stressors. Peripheral neuropathy, disfunction of nerve cells outside of the brain and spinal cord (CNS), is very common and found in most serious diseases in some form. Neuropathy due to physical trauma most commonly due to abnormal pressure on a nerve at locations of tunnels between bone, ligaments and muscle tendons (such as carpal tunnel syndrome due to repetitive use). A blow to the ulnar nerve at the groove of the humerous at the elbow results in tingling, numbness and pain and is known as "hitting my funny bone", a form of direct trauma. Abnormal bone growth or tumors will also constrict the openings that nerves travel and cause neuropathy.

At least half of the people with diabetes will develop some form of neuropathy that may even lead to eventual amputation of toes and fingers or limbs. Guillian-Barre' syndrome (nerve de-myelination) most often occurs after an infection, surgery or immunization and disrupts the nerve impulse. Leprosy is the most common bacterial cause of neuropathy and Herpes is the most common viral cause. Autoimmune disorders such as Systematic Lupus Erythematosis, Rheumatoid Arthritis, Polyarteritis Nodosa, and Sjogren Syndrome are the cause of neuropathy and add to the suffering of those affected. Two inherited disorders are known to cause neuropathy: CharcotMarie-Tooth Disease and Friedreich's Ataxia.

The B-vitamins (especially B-12) and folate seem to play a crucial role in the prevention of neuropathy as deficiencies in these vitamins result in systemic (body wide) neuropathic symptoms. Exposure to toxic substances is a very frequent cause of neuropathy and a huge problem in the chemical, petroleum, mining and manufacturing industries. The causative agents are organic solvents, organic base materials, welding fumes and heavy metals (cadmium, lead, arsenic, mercury and hexavalent chromium). Pharmaceutical drugs, especially drugs of chemotherapy, are known potent causes of neuropathy. Certain drugs interfere with nerve cell metabolism directly and others are more systemic in their effects. For drug-induced neuropathy, taking L-acetyl-carnitine (available at any vitamin store) may be helpful.

Mild polyneuropathy may develop in persons with low thyroid hormone levels. Individuals with abnormally enlarged skeletal extremities (acromegaly), caused by an overabundance of growth hormone, may also develop mild polyneuropathy.

\section{Discussion}

The causes of the neuropathy are such as to interfere with the natural inherent rhythms and cellular physiology of nerves. The first means that organic life used to signal changes in cellular response in order to bring the cell or organism into resonance with the totality of the living environment was chemical or, in the case of living cells and organisms, biochemical. The second was the development of specialized cells known as nerves in the more complex animate organisms. Both the biochemical and nervous systems are scalar phenomena working at very minute receptors on cellular membrane surfaces to elicit first cellular effects, then localized tissue effects and eventually to organismal effects. Ultimately, all the bewildering arrays of the systems of cell physiology come into play. Cellular surface phenomena play a crucial role in this drama. Biochemical integration is by nature slower and more whole-body pervasive than nervous integration while nervous integration is much more localized and much faster. Thus, the effects of hormones (chemical) are gradual in intensity and ebb just as slowly, like a sine wave. Conversely, the effects of nerve stimulation are, in comparison, very fast and almost instantaneous.

Cellular surface phenomena entail many phenomena such as transport of nutrient and waste substances, reception of signal molecules, release of specific substances, cellular movement, transmembrane flux of ions, cellular adhesion and the integration of the extracellular matrix (ECM) with cellular architecture, just to name a few. Many enzyme systems within the outer cell membrane system are sensitive to toxic compounds such as heavy metal ions, man- 
made organic industrial compounds and pharmaceuticals. All cells but especially nervous cells are very sensitive to ischemia - the deprivation of oxygenated blood and interstitial fluid. Nerve cells have a great need for energy as adenosine tri-phosphate (ATP) and thus require increased oxygen levels more than other cells, as cell surface phenomena requires great amounts of ATP in order to function. Since nerve cells have very long processes that are far removed from the nerve cell body, anything that interferes with cellular transport from the cell body to the synapse and return will have detrimental effects on nerve cell function. Neurotransmitters are manufactured in the cell body and transported for use to the synaptic region at the end of the nerve cell axon. Also, events that occur at the synapse are sensitive to toxins that degrade key enzyme systems involved with neurotransmitter events. Since the nerves that go to and come from the extremities are the longest nerves of the body, these nerves are usually affected first. This one of the reasons that these conditions are called "peripheral" neuropathy.

Both the biochemical and nervous system modes of the integration of bodily events in time are ultimately dependent upon the neuroendocrine system to set the pace and rhythms of cellular metabolism throughout the body. Thus, anything that disrupts the neuroendocrine system of the CNS affects the entire organism. The peripheral nervous system coordinates all events in the body proper. The extensive network of peripheral nerves includes the motor nerves (that help your muscles contract) and the sensory nerves (that allow for the range of feeling of sensations). In addition, your peripheral nerves help to control the involuntary functions of the autonomic nervous system that regulates your internal organs (and their products), the sweat glands and rheostatic (a more proper term than homeostatic) mechanisms.

Unfortunately, peripheral nerves are fragile and easily damaged as described. Damage to a peripheral nerve interferes with communication between the area of service and the CNS that in turn affects the ability to move certain muscles or feel normal sensations. The symptoms will depend upon the cause of the neuropathy and upon the nerve(s) involved. More importantly are the effects that neuropathy has on the local body environment in the roll of recovery from trauma. The blood distribution system is such that a vessel is no farther than 3 cells away from any given cell. Thus, neuropathy can also result from severe vasculitides, a group of disorders in which blood vessels are inflamed. When the blood vessels are inflamed or damaged, blood supply to the nerve can be affected, injuring the nerve. Nerve cell axons travel in bundles called nerves. Peripheral cell bodies are located in the skin, muscle spindle fibers, Golgi tendon complexes, nerve ganglia and various tissues that are all served by the blood distribution system. Nerves control the amount of blood flow to the tissues of the body. Trauma to the blood vessel sets in motion a series of events that results in the production of helpful and pathological products that must then be eventually removed from the tissues by elements of the immune system. The immune system cells and the nerve cells use the same compounds, called cytokines, to communicate with each other as well as among themselves. Damage to nerve cell membrane surface recetors due to toxic substances will interfere with this communication. The liver is responsible for most of the non-cellular elements of blood serum (the liquid containing the blood cells). These elements are kept static until trauma initiates events that result in the helpful and pathological products. The only active cellular components are the nerve cells and the immune cells. It is the pathological products (cellular debris, foreign body debris, plaque, non-functional ECM components, displaced blood proteins, dissolved gases $\{$ low $\mathrm{O} 2$, high $\mathrm{CO} 2\}$ and damaged enzyme systems; to name a few) that are the additional cause of neuropathy. The peripheral nerve cells of the body do not replace themselves whereas the immune cells are replaced continuously.

Thus, neuropathy is a prime candidate for the common link between Western and Eastern medicine. The etiology of disease in Eastern medicine is essentially the same as that of Western medicine. However, the philosophical concepts are seemingly quite different. On the surface this seems irreconcilable. It is with peripheral neuropathy that the common ground between these two systems of thought is revealed.

So, just what is this western concept of peripheral neuropathy? Peripheral neuropathy describes damage to the peripheral nervous system (PNS). The PNS transmits information from the brain and spinal cord (the CNS) to every other part of the body. Peripheral nerves also send sensory information to the brain and spinal cord from the skin and tissues. Damage to the PNS interferes with this communication system. Like static on a radio, peripheral neuropathy distorts and sometimes interrupts messages between the brain and the rest of the body. Neuropathy may affect the nerve directly or impair the synapse or neuromuscular junction, depending upon the cause. The neuropathy may be symmetrical, generalized or focal (a specific location). Generalized peripheral neuropathies are symmetrical, and usually due to various systematic illnesses and disease processes that affect the PNS in its entirety. There are three categories of generalized neuropathy: distal axonopathy, myelinopathies and neuronopathies.

Distal axonopathies result from some metabolic or toxic derangement of neurons. These may be caused by metabolic diseases such as diabetes and renal failure, deficiency syndromes (such as malnutrition and alcoholism), or the direct effects of toxins or drugs.

Myelinopathies are due to a primary attack on myelin (cells that wrap around and cover nerve cells) causing an acute failure of impulse conduction. The most common cause is acute inflammatory demyelinating polyneuropathy (AIDP or Guillain-Barré syndrome), though other causes include chronic inflammatory demyelinating syndrome (CIDP), genetic metabolic disorders, or toxins.

Neuronopathies are the result of destruction of peripheral nervous system (PNS) neurons. They may be caused by motor neurone diseases, sensory neuronopathies (e.g., Herpes zoster), toxins or autonomic dysfunction. Neurotoxins may cause neuronopathies, such as the chemotherapy agent vincristine.

Because every peripheral nerve has a highly specialized function in a specific part of the body, a wide array of symptoms can occur when nerves are damaged. Some people may experience temporary numbness, tingling, and pricking sensations (paresthesia), sensitivity to touch, or muscle weakness. Others may suffer more extreme symptoms, including burning pain (especially at night), muscle wasting (atrophy), paralysis and organ or gland dysfunction. People may become unable to digest food easily, maintain safe levels of blood pressure, sweat normally, or experience normal sexual function. In the most extreme cases, breathing may become difficult or organ failure may occur. Impaired function and symptoms depend on the type of nerves (motor, sensory, or autonomic) that are damaged. Motor nerves control movements of all muscles under conscious control, such as those used in walking, grasping things, or talking. Sensory nerves transmit information about sensory experiences, such as the feeling 
of a light touch or the pain resulting from a cut. Autonomic nerves regulate biological activities that people do not control consciously, such as breathing, digesting food, and heart function.

If only a single nerve is affected, it is called mononeuropathy. If multiple nerves affecting all extremities are affected, it is called polyneuropathy. Sometimes one or more isolated nerves that affect different areas within the body occur. This is called mononeuritis multiplex. In acute neuropathies, symptoms appear suddenly, progress rapidly, and resolve slowly as damaged nerves heal. In chronic forms of neuropathy, symptoms begin subtly and progress slowly. Some people may have periods of relief followed by relapse. Others may reach a plateau stage where symptoms stay the same for many months or even years. Some chronic neuropathies may worsen over time, but very few forms prove fatal unless complicated by other diseases. Occasionally the neuropathy is a symptom of another disorder.

In the most common forms of polyneuropathy, the nerve fibers most distant from the brain and the spinal cord malfunction first. Pain and other symptoms often appear symmetrically, for example, in both feet followed by a gradual progression up both legs. Next, the fingers, hands, and arms may become affected, and symptoms can progress into the central part of the body. For example, many people with diabetic neuropathy experience this pattern of ascending nerve damage and accompanying altered function.

Symptoms are related to the type of nerve affected and may be seen over a period of days, weeks, or years. Muscle weakness is the most common symptom of motor nerve damage. Other symptoms may include painful cramps, spasms and fasciculations (uncontrolled muscle twitching visible under the skin), muscle loss (atrophy), bone degeneration, and changes in the skin, hair, and nails. These more general degenerative changes also may result from sensory or autonomic nerve fiber loss.

Sensory nerve damage causes a more complex range of symptoms because sensory nerves have a wider, more highly specialized range of functions. Larger sensory fibers enclosed in myelin (a fatty protein sheath that coats and insulates many nerves) register vibration, light touch, and position sense. Damage to large sensory fibers lessens the ability to feel any vibrations and touch, resulting in a general sense of numbness and tingling, especially in the hands and feet. People may feel as if they are wearing gloves and stockings even when they are not. Many patients cannot recognize by touch alone the shapes of small objects or distinguish between different shapes. This damage to sensory fibers contributes to the loss of reflexes (like motor nerve damage). Loss of position sense often makes people unable to coordinate complex movements like walking, fastening buttons, or in maintaining their balance when their eyes are shut. Neuropathic pain is difficult to control and can seriously affect emotional well-being and overall quality of life. Neuropathic pain is often worse at night, seriously disrupting sleep and thus adding to the emotional burden of sensory nerve damage.

Smaller sensory fibers without myelin sheaths transmit pain and temperature sensations. Damage to these fibers interferes with the ability to feel pain or changes in temperature. These nerves may also be the origin of a burning pain sensation. Eventually, people may fail to sense that they have been injured from a cut or that a wound is becoming infected. Others may not detect pains that warn of impending heart attack or other acute conditions. Pain receptors in the skin can also become oversensitized, so that people may feel severe pain from stimuli that are normally painless. A generalized body pain in the muscles without apparent cause is termed fibromyalgia.

Symptoms of autonomic nerve damage are diverse and depend upon which organs or glands are affected. Autonomic nerve dysfunction can become life threatening and may require emergency medical care in cases when breathing becomes impaired or when the heart begins beating irregularly. Common symptoms of autonomic nerve damage include an inability to sweat normally (that may lead to heat intolerance), lack of tears, saliva and digestive fluids, a loss of bladder control (that may cause infection, hesitance or incontinence), impotence/infertility and an inability to control muscles that expand or contract blood vessels in order to maintain safe blood pressure levels. A loss of control over blood pressure can cause dizziness, blurred vision, lightheadedness, or even fainting when a person moves suddenly from a seated to a standing position. Gastrointestinal symptoms frequently accompany autonomic neuropathy. Nerves controlling intestinal muscle contractions often malfunction, leading to diarrhea, constipation, incontinence, abdominal bloating, early satiety and unintentional weight loss. Many people also have problems eating or swallowing if certain autonomic nerves are affected.

Now that we have a background in peripheral neuropathy, the discusion will shift to that of Traditional Chinese Medicine.

Traditional Chinese Medicine (TCM) recognizes a series of syndromes that tie into the neuropathy of the Autonomic Nervous System of Western medicine exceedingly well. Western medicine does not have a philosophy or generalized theory of health and wellbeing, as does Eastern medicine. Western medicine is totally scientific and wholly left-brain in outlook. Eastern medicine is several millennia old, based upon thought derived from meditational practice called Qi Gong and many centuries empirical clinical practice. Thus, it is both left-brain analytical and right-brain conceptual. What worked and what did not work got passed on. In ancient China, you paid the doctor to keep you well. If you got sick, you changed doctors.

TCM delineates four major means of identifying disease patterns. These are 1. The Eight Principle Disease Patterns, 2. the Qi \& Blood Pattern, 3. The Organ Pattern and 4. The Disease Evil Pattern. The Disease Evil Pattern is further broken down into two systems: The 6 Channel Pattern and the Four Aspect Pattern. Each will be briefly described in turn.

The Eight Principle Pattern deals with polar opposites as the ideal.

These are External: Internal, Cold: Heat, Excess: Deficiency and Yin: Yang. It is noted from clinical experience that the true idealized opposite is rarely if ever seen. The External: Internal mode deals with whether the disease has an origin that is external to or internal to the body. External deals with pathogens from the environment invading the body, whatever the cause. Internal deals with derangements from rheostasis and meridian/organ problems, which are the origins of disease. The Cold: Heat mode deals with whether the disease is such as to cause internal coldness or internal heat within the body as a whole or in part. The Excess mode refers to whether or not the body is in a condition that tends to release energy, produces heat or cold within the body and causes some form of stasis or congestion. The Deficiency mode refers to whether or not the body is in a condition that tends to use up energy, produce cold signs, reduces organ function and denotes reduced bodily fluids. The concept of Yin: Yang is that of opposites. Yin refers to internal, cold, deficiency and feminine. Yang refers to external, heat, excess and masculine. Yin refers to the substance(s) 
of the body while Yang refers to the energies and functions of the body. These eight principles are in constant dynamic flux with all the possible combinations yielding a set of signs and symptoms at any point in time that indicate the overall condition of the body.

Let move this scheme into the realm of modern Western physiology. You must remember that ancient peoples did not have the benefit of modern technology and concepts that arise from that modern technology and the knowledge that it yields. A very good review of the 8 Principal Disease Patterns may be found here (A) and here (B).

\section{External: Internal}

\section{External}

This is not difficult to understand from a physical viewpoint but is more difficult to imagine from an energetic/field viewpoint. Modern science has given us remarkable detail of the workings of cellular physiology, organ physiology and an integrated overall body physiology. Any online search will yield a plethra of information. However, if you want to discover information about the energetic/ field interactions of the Human Body-Field with other energy/field systems encountered within the local environment, you are relegated to the "fringe" of what is called alternative and complementary medicine. This is because the modern scientific commulity refuses to admit to the existance of such phenomena! This fact is what divides the so called modality of Western medicine from that of Homeopathy, Naturopathy and Acupuncture/Oriental Medicine. ${ }^{3}$

It is well known that an energetic shift always precedes a physiological reaction by cellular metabolism. There is a plethra of adverse electromagnetic and geomagnetic phenomena in the local environment to cause distress in a healthy organism. Early Chinese medical theory in its ignorance had attributed some external causes of disease to be of negative spiritual origin. While it is true that the negative intent of other individuals and the influence of spirit entities upon the mental/emotional aspects of human consciousness may cause the affected individual to suffer mentally/emotionally generalized trauma, the direct trauma with environmental aspects is usually geomagnetic in ancient times and electromagnetic as well as geomagnetic in our modern times.

The whole idea of feng shui and dowsing a site for construction nuances have their roots in the effects that negative geomagnetic anomalies have upon the wellbeing of individuals. Today, we need to be aware of the effects of the myriad of man-made elecromagnetic frequencies have upon the Body-Field of an indiviual. This includes both the electric component and the magnetic component. In fact, derangements caused by the magnetic component are much more detrimental and longer lasting!

Physical trauma is easily understood from the point of view of direct effects to organ tissue, blood vessels and nerves. The subsequent blood element involvement in the affected area is inflammation and the response of white blood cells, mast cells and tissue fibroblasts.

The other direct trauma is that caused by the various pathogenic microorganisms and multicellular parasites. Usually parasites cause a type of chronic debilitation that is not usually directly life-threatening. However, pathogenic microorganisms may overwhelm the immunologic capability of an individual and cause death. The mode and route of infection has a lot to play in this drama. Parasites and pathogenic microorganisms may cause any number of neuropathies.

\section{Internal}

As Internal deals with derangements from rheostasis and meridian/ organ problems, which are the origins of disease, anything that disrupts the smooth flow of energy, nutrients and fluids will cause some form of neuropathy. Nerves are physical structures that have an origin in 1 place, a usually long axonal component and a nerve ending in another place. Thus, the neuron is susceptible to trauma at a number of points along its cellular perimeter that are not at any 1 locale and may be quite a distance apart. Cell surface structure turnover is not rapid and thus components of the cellular membrane are exchanged/ repaired slowly. It usually takes at least 3 days for complete turnover to occur. Thus, any chronic trauma will eventually overwhelm the capacity for the neuron to repair itself and result in some form of peripheral neuropathy.

One of the most pervasive aspects of any internal cause is that of a genome with characteristics that yield such issues as multiple sclerosis and other nervous tissue related anomalies. This form of chronic disease is outside the preview of this article.

\section{Cold: Heat}

The hypothalamus is the nervous locus that regulates the rheostatic temperature of the body. The thyroid gland regulates the overall basal metabolic rate (BMR) of the body. Blood borne cytokinins released by immunological cell types alert the hypothalamus to the invasion of pathogenic microorganisms. The hypothalamus then sets the upper temperature of the body by 2-3 degrees as this is too high for the invading pathogens and the body can operate at this temperature for a short time without undue effects.

Some microorganisms and parasites seem to be able to circumvent, to a great extent, the normal onset of immune responses. This causes a debilitation in the area of infection in terms of tissue damage and the disruption of blood flow. Since inflammation is reduced or absent, the tissue appears pale and feels cold. One example is that of leprosy.

\section{Excess: Deficiency}

This is means of describing the relative state of the energy field parameters of the body and the ability of the qi of the body to respond to stressors.

\section{Yin:Yang}

In this instance, Yin refers to the actual substances used by the body (nutients and fluids) while Yang refers to the metabolic capability of the body.

\section{The Qi \& blood pattern}

The Qi \& Blood Pattern denotes the condition and disharmonies of the flow of energy and blood (and in some part fluids) throughout the body. The normal functioning of the organs within the body is responsible for the production of Qi (energy) \& Blood (fluids). The Qi $\&$ Blood are required for the normal functioning of the organs. Thus, anything that disrupts either the organs or the Qi \& Blood will have a general debilitating effect within the body. Thus, with this system, both the organs and the area of disruption are taken into account. The abnormal conditions of Qi are deficiency and stagnation. Qi deficiency goes hand in hand with organ hypofunction. Qi stagnation is caused by mental \& emotional disturbances, dietary irregularities, invasion of external pathogens and trauma. The signs of Qi stagnation 
are distending pain and the sensation of oppression. The abnormal conditions of Blood are deficiency, stagnation and heat. Blood deficiency refers to any insufficiency condition of the blood due to lack of production or loss of blood due to trauma. Blood stagnation refers to a condition of restricted blood flow caused by trauma, Qi deficiency/stagnation and blood heat. Signs of blood stasis are stabbing pain, swelling, bleeding and purple bruises, macules/rashes and spots. Blood Heat occurs during the contraction of a febrile disease. Blood Heat causes internal bleeding, bleeding from orifices and the skin, red papules and macules, thirst and a rapid pulse. Qi stagnation and Blood stasis usually occur together and Qi deficiency and Blood deficiency together are common.

\section{The organ pattern identification}

The purpose of Organ Pattern identification is to determine what $\operatorname{organ}(\mathrm{s})$ is affected by disease and to identify their morbid changes. Please remember that the ancient Chinese did not have the benefit of modern science when they developed their concepts of medicine based upon observation, gross anatomical autopsy and clinical practice. The TCM system of meridians and organs is in great part conceptual. It is quite amazing that the ancient Chinese were able to develop a medical system that works so well. Disorders of Heart Yin, Yang, Qi and Blood are characterized by disturbances in blood flow, mental disturbances and emotional disturbances. Disturbances of Lung Qi result in susceptibility to external pathogens (viral and bacterial infections), production of cough, shortness of breath and weakness. Lung Yin deficiency causes dry mouth $\&$ throat, dry cough and enduring Lung infections. Diseases of the Spleen, Stomach and the Intestines involve problems of food digestion and assimilation, distribution of nutrients and excretion of solid wastes. Impairment of the Spleen results in digestive problems, the accumulation of water-dampness and blood management failure. Impairment of the Stomach results in damp turbidity food accumulations. Impairment of the Intestines results in diarrhea or constipation. Liver \& Gallbladder diseases result from the binding suppression of Liver Qi, which manifests as superabundance, and problems in the storage of blood (55\% of the body's blood is stored in the Liver, when at rest). Liver/Gallbladder binding depression results in mental depression, bile secretion disorders, ulcers, menstrual irregularities and Liver/Gallbladder channel disorders. Liver blood vacuity results in expectoration of blood, nosebleeds and profuse menses. Liver Qi superabundance results in severe emotional disturbances, mental agitation, pronounced heat signs in the upper part of the body, nausea \& vomiting, irritability and sensory organ disorders. Kidney and Urinary Bladder diseases manifest as body fluid disorders, reproductive disorders, bladder disorders and generalized fatigue.

\section{The disease evil pattern identification}

Disease Evil Pattern identification deals with the determination of which external pathogen is the cause of the disease. These causes are Wind, Cold, Heat, Summer Heat, Dampness and Dryness. In addition, dietary irregularities, phlegm and blood stasis are involved. In these cases, it is the result in the alteration of normal physiological function that is the basic problem. This in turn causes disruption of the internal milieu and loss of rheostasis within the body, which is the origin of some form of disease.

In case, Eastern or Western medicine, it is my thesis that disease is first a problem of body energy field resonance disharmony followed by some form of neuropathy and finally the signs and symptoms of disease. The form of the neuropathy may be:

A. Interference in normal nervous element physiology and the resulting breakdown of rheostatic functions within the body that are directly coordinated by the elements of the central and peripheral nervous systems

B. Failure of the coordinated release of neuropeptides by the PinealHypothalamic-Pituitary axis that eventually affect the stimulation of hormonal tissues outside of the CNS, which indirectly leads to the breakdown of rheostatic functions (or a combination of both, which is more likely).

C. The almost bewildering array of disease symptoms lets us know that the coordination of rheostasis involves all the tissues and organs of the body in some manner. So now let us review Western neuropathy, TCM disease differentiation and then compare the two schemes.

\section{Western neuropathy'}

A. If a sensory nerve is damaged, symptoms may include:

a. Pain: burning, dull, sharp, jabbing or electric-like

b. Numbness, tingling, "pins \& needles" \& itching

c. Loss of feeling

d. The sensation that you're wearing an invisible glove or sock

e. Extreme sensitivity to touch, even light touch

B. If a motor nerve is damaged, symptoms may include:

a. Lack of coordination, falling over, lack of dexterity

b. Partial or complete loss of movement

c. Muscle atrophy \& bone degeneration

d. Cramping and spasms, tremors

e. Difficulty in swallowing or breathing

C. If an autonomic nerve is damaged, symptoms may include:

a. Blurred vision, dizziness, fainting due to inability to control blood pressure

b. Decreased ability to sweat \& intolerance to heat

c. Intolerance to cold

d. Abdominal bloating, nausea \& vomiting after meals, early satiety

e. Diarrhea and/or constipation

f. Unintentional weight loss (greater than $5 \%$ )

g. Urinary incontinence, feeling of incomplete bladder emptying, urinary hesitancy

h. Impotence and infertility

\section{Other complications may include:}

a. Ischemia (decreased oxygen/decreased blood flow)

b. Frostbite (prolonged exposure to cold) 
c. Systemic or metabolic disorders, poor wound healing

d. Infectious or inflammatory conditions

e. Depression \& insomnia

\section{TCM disease differentiation ${ }^{2}$}

i. Excess or Deficiency, Internal or External, Heat or Coldness.

ii. Yin (substance) or Yang (function).

iii. Qi (stagnation, deficiency), energy for function.

iv. Blood (stagnation, deficiency, heat) .

v. Organ (Qi deficiency, Blood deficiency, Yin deficiency, Yang deficiency, hyperactivity, Fire flaming upward, counter flow depending upon the particular organ).

vi. Disease Evils (Wind, Coldness, Heat, Fire, Summer Heat, Dampness, Dryness, Phlegm, Food stagnation and combinations).

\section{Scheme comparison}

First we need to realize that the TCM disease differentiations describe patterns of signs and symptoms. These patterns have associated with them a number of aberrations from normal physiology, function and metabolic products. All the TCM disease pattern aberrations listed are exactly those as described as the result of sensory, motor and autonomic nerve neuropathy according to the TCM channel, organ and combinations thereof involved. Thus, I make the statement that neuropathy is the common ground between Western medicine and TCM.

The Mode Excess and Deficiency is self-explanatory, as are Internal and External as to origin. The Heat mode details the condition of higher temperatures within the body as the result of pathogenic influences and excess functionality. The Cold mode details the condition of lower body temperatures as the result of pathogenic influences and reduced functionality. In both cases, the effect need not be whole body and may involve regional or local areas only.

In my opinion, in order to understand the Yin:Yang mode, we need to consider the autonomic nervous system (ANS). Yin is associated with night, reception and substance while Yang with day, action and function. Yin and Yang are said to change in the body in cycles and have within each, elements of the other. This in part describes the action of the sympathetic and parasympathetic components of the ANS. The sympathetic nervous component deals with being active, speeds up functionality, moves the blood to the muscles and surface of the body and sharpens awareness. The parasympathetic nervous component deals with recuperation, slows functionality, moves blood to the viscera of the body and puts one to sleep. Yang uses energy and substance while Yin generates energy and substance. Thus, any neuropathy in the nerves of the ANS will disrupt the balance of Yin and Yang in the body as evidenced by deranged rheostatic functionality, i.e., loss of control of blood pressure, sleep problems, digestive disorders and bowel problems.

I make the additional connection between the embryonic tissue layers and the Yin:Yang mode. The ectoderm gives rise to the skin and nervous system. The endoderm gives rise to the mucosa and digestive system. Both layers contribute to the mesoderm that gives rise to the skeletal and muscular systems. I propose that this is the ultimate origin of the mode that TCM has designated as Yin and Yang, Yin for the parasympathetic and digestive function and Yang for the sympathetic and nervous function. Both sets of the ANS send nerves to the tissues derived from the mesoderm.

Qi and Blood function very closely together but one may ostensively be deranged without the other. The blood carries oxygen and glucose to every part of the body such that every cell is, at most, only 3 cells away from a blood vessel. The exception is the CNS in which there are no blood vessels at all. Blood also carries proteins and enzymatic cofactors that will cause tissue inflammation and swelling upon bleeding outside of the vessels into the tissue. This will cause a reduction of oxygen and glucose availability for cells in the area that directly reduces cell, tissue and organ functionality. This will cause Qi and Blood stagnation and then results in Qi deficiency, i.e., reduced functionality, and even cell death if severe enough. ${ }^{3-5}$

The TCM organ systems are tied to the TCM meridian system but each has functionality apart from those held in common. A lack of organ functionality affects the meridian and a blockage in the meridian has consequences in organ functionality. Since the ANS coordinates the functionality of the viscera (internal organs), any disease in the organs will eventually affect the meridian system. Any disruption in the natural flow of energies in the meridian system will also affect the organ associated with the blocked meridian with characteristic signs of those of neuropathy.

\section{Conclusions}

The above information presented demonstrates quite well that neuropathy is at the heart of both TCM and a large component of Western medicine.

\section{Acknowledgments}

None.

\section{Conflicts of interest}

Author declares there are no conflicts of interest towards the article.

\section{Citations}

A. http://www.shen-nong.com/eng/exam/diagnosis eightprinciples.html

B. https://www.sacredlotus.com/go/diagnosis-chinese-medicine/ get/differentiation-syndromes-8-principles-ba-gong-tcm

\section{References}

1. Latov N. Peripheral Neuropathy: When the Numbness, Weakness, and Pain Won't Stop. New York, USA: Demos Medical Publishing; 2006.

2. Wiseman N, Ellis A. Fundamentals of Chinese Medicine, Revised Edition, Brookline, Massachusetts, USA: Paradigm Publications; 1996.

3. Block EF. The Human Body-Field. Journal of Informational Medicine. 2009.

4. Block EF. Useful Conceptual Constructs In The Clinical Application Of Informational Medicine. Journal of Informational Medicine. 2011.

5. Sobel E, Dunn M, Davanipour Z, et al. Elevated risk of Alzheimer's disease among workers with likely electromagnetic field exposure. Neurology. 1996;47(6):1477-1481. 